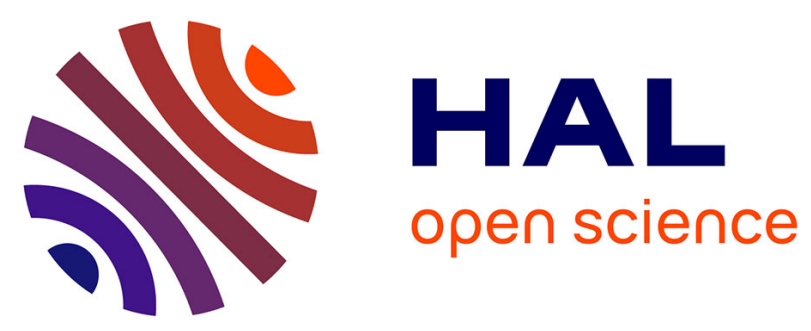

\title{
Home-based exercise in autoimmune myasthenia gravis: A randomized controlled trial
}

\author{
S Birnbaum, R Porcher, P Portero, B Clair, S Demeret, B Eymard, M
} Gargiulo, E Louët, Sonia Berrih-Aknin, R Le Panse, et al.

\section{- To cite this version:}

S Birnbaum, R Porcher, P Portero, B Clair, S Demeret, et al.. Home-based exercise in autoimmune myasthenia gravis: A randomized controlled trial. Neuromuscular Disorders, 2021, 31 (8), pp.726-735. 10.1016/j.nmd.2021.05.002 . hal-03442076

\section{HAL Id: hal-03442076 \\ https://hal.science/hal-03442076}

Submitted on 7 Dec 2021

HAL is a multi-disciplinary open access archive for the deposit and dissemination of scientific research documents, whether they are published or not. The documents may come from teaching and research institutions in France or abroad, or from public or private research centers.
L'archive ouverte pluridisciplinaire HAL, est destinée au dépôt et à la diffusion de documents scientifiques de niveau recherche, publiés ou non, émanant des établissements d'enseignement et de recherche français ou étrangers, des laboratoires publics ou privés. 


\title{
Home-based exercise in autoimmune myasthenia gravis: A randomized controlled trial
}

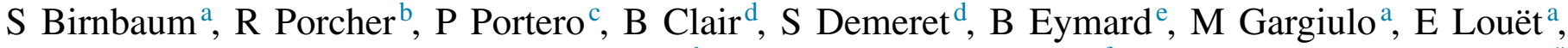 \\ S Berrih-Aknin ${ }^{\mathrm{a}}$, R Le Panse ${ }^{\mathrm{e}}$, P Aegerter ${ }^{\mathrm{d}}$, JY Hogrel ${ }^{\mathrm{a}}$, T Sharshar ${ }^{\mathrm{f}, *}$, the MGEX Study Group ${ }^{\#}$ \\ anstitute of Myology, Paris, France \\ ${ }^{\mathrm{b}}$ Hotel-Dieu Hospital Clinical Epidemiology Centre, Paris, France \\ ${ }^{\mathrm{c}}$ Université Paris-Est, Creteil, France \\ ${ }^{\mathrm{d}}$ APHP, Assistance Publique - Hopitaux de Paris, France \\ ${ }^{\mathrm{e}}$ Paris Descartes University Institute of Psychology, Paris, France \\ ${ }_{\mathrm{f}}^{\mathrm{f}}$ GHU-Psychiatrie \& Neurosciences site Sainte-Anne 75014 Paris, France
}

\begin{abstract}
The tolerance of exercise and its effects on quality of life in myasthenia gravis are not currently backed up by strong evidence. The aim of this study was to determine whether exercise as an adjunct therapy is well tolerated and can improve health-related quality of life (HRQoL) in stabilized, generalized autoimmune myasthenia gravis (gMG). We conducted a parallel-group, multi-center prospective RCT using computer-generated block randomization. Adults with stabilized, gMG, and no contra-indication to exercise, were eligible. Participants received usual care alone or usual care and exercise. The exercise intervention consisted of 3-weekly 40 min sessions of an unsupervised, moderate-intensity home rowing program over 3 months. The primary endpoint was the change in HRQoL from randomization to postintervention. Assessor-blinded secondary endpoints were exercise tolerance and effects on clinical, psychological and immunological status. Of 138 patients screened between October 2014 and July 2017, 45 were randomly assigned to exercise $(n=23)$ or usual care $(n=20)$. Although exercise was well tolerated, the intention-to-treat analysis revealed no evidence of improved HRQoL compared to usual care (MGQOL-15-F; mean adjusted between-groups difference of -0.8 points, 95\%CI -5.4 to 3.7). Two patients hospitalized for MG exacerbation were from the usual care group.
\end{abstract}

(C) 2021 Elsevier B.V. All rights reserved.

Keywords: Myasthenia gravis; Exercise; Randomized controlled trial; Autoimmune disease; Quality of life.

\section{Introduction}

Myasthenia gravis (MG), a rare autoimmune disorder of the neuromuscular junction is characterized by fluctuating weakness involving variable combinations of ocular, bulbar, respiratory and limb muscles. Treatment of MG primarily relies on immunosuppressive therapies, notably the association of corticosteroids with non-steroidal immunosuppressive agents [1]. These therapeutic strategies aim to reduce symptoms, improve functional status and

\footnotetext{
* Corresponding author.

E-mail address: tsharshar@gmail.com (T. Sharshar).

\# Participants are listed at the end of this article.
}

health-related quality of life (HRQoL); three dimensions which can be assessed with specific scores [2]. Despite appropriate therapeutic management, individuals with MG still have reduced HRQoL which is not improving despite therapeutic advances [3]. Therefore, adjuvant therapy for improving HRQoL in MG is warranted. Whilst rest is indicated during an exacerbation, exercise may be a relevant option in stabilized MG as it targets muscle-related symptoms, i.e. weakness and fatigability.

Exercise therapy has been shown to improve strength and functional capacity in various neuromuscular diseases such as facioscapulohumeral disease [4] and other muscular dystrophies [5]. There are physiological and clinical arguments for its therapeutic use in MG. It induces 
remodeling of the neuromuscular junction in experimental models [6] and interventional human studies have shown improved lower limb strength [7,8], walking capacity and overall function [9-11] and, exercise self-efficacy [11]. However, these studies were non-randomized [7-9,11] and included small numbers [7-11].

In addition, exercise has positive psychological and immunological effects, which are of potential benefit to individuals with MG. Symptoms of depression and anxiety have been shown to decrease with exercise in chronic illness [12-14]. Favorable immunomodulatory effects have been induced by the release of myokines during repetitive muscle contractions $[15,16]$. Because of its pluripotent effects, it is conceivable that exercise could improve HRQoL in MG. The objective of this study was to evaluate whether an unsupervised home exercise program (ExP) could improve MG-related HRQoL of individuals with stabilized and gMG, with reduced HRQoL. Secondary objectives were to assess exercise tolerance and the impact of exercise on physical, psychological and immune status.

\section{Methods}

\subsection{Study design}

We conducted a two-arm, parallel group, multi-center randomized controlled trial (RCT) comparing a home-exercise program to usual care (UC). Full details of the study rationale, design, methods and statistical analysis have been previously published [17]. The overall study duration for an individual participant was 36 weeks. Total intervention time was 12 weeks beginning at randomization at month 3 (M3), ending at month 6 (M6). This was followed by a 3-month followup period (with no intervention), ending at month 9 (M9). Writing of this manuscript follows the CONSORT guidelines [18]. Ethics approval to carry out the study on human subjects was granted by the French regulatory board (Comité de Protection des Personnes Ile de France XI under the CPP number \#13064 on 13/12/2013 and authorized by the Agence National de Sécurité du Médicament et des produits de santé on 11/10/ 2013). The trial was conducted in accordance with the Helsinki Declaration and was prospectively registered in ClinicalTrials.gov (NCT02066519).

\subsection{Participants and setting}

Three specialized neuromuscular centers in France screened patients with autoimmune MG during regular outpatient consultations and study information was diffused to MG-patient groups. Patients aged 18-70 years with a confirmed diagnosis of mild-moderately-severe, gMG (class II-III according to the Myasthenia Gravis Foundation of America (MGFA) classification) were eligible. Six months of MG disease stability, determined by the treating neurologist, and reduced HRQoL (MGQOL-15-F score $\geq 15$ ), was required for inclusion. Exclusion criteria included any contraindication to exercise which precluded participation in the experimental arm, other neuromuscular pathology, disabling rheumatological or orthopedic condition, chronic pain, recent hospitalization for a serious medical or surgical condition, anemia (hematocrit $<30 \%$ ), pregnancy or severe cognitive impairment necessitating specific protection (Table S1). Written, informed consent was obtained from all participants prior to enrolment.

\subsection{Randomization, allocation concealment, blinding}

Following the 3-month evaluation, if MG stability was confirmed, consecutive participants were randomized to either the intervention (UC+exercise) or control (UC) group with a 1:1 allocation ratio as per a computer-generated randomization schema stratified by center using permuted blocks of randomly varying sizes. To ensure concealment, block sizes were not disclosed. The randomization list was constructed prior to the beginning of the study by a statistician with no clinical involvement in the study. Group allocation was revealed exclusively to the non-blinded physiotherapist at the moment of randomization (M3), via a software (CleanWeb), and was concealed until the moment of group allocation. Investigators, evaluators and study personnel were all masked to group assignment for the duration of the study. Only the physiotherapist performing the neuromuscular evaluations and managing the training program was aware of group allocation.

\subsection{Intervention}

All participants received UC and evaluations as per the study protocol. The control group were instructed to maintain their regular physical activity without starting a new exercise program or increasing baseline activity. Baseline activity was measured over one week, prior to randomization, using a triaxial accelerometer (Movemonitor, McRoberts, Netherlands). The intervention group was invited to participate in a 12week home-based aerobic exercise program (ExP) using a rowing ergometer $\left(\right.$ Concept $2^{\mathrm{TM}}$ ), Table S2. The ExP consisted of three $40 \mathrm{~min}$ sessions per week, i.e. a total of 36 sessions over the intervention period. A chest heart rate (HR) monitor (Garmin $®$ ) was worn by participants and training HR was visualized on a screen for direct feedback. Each $40 \mathrm{~min}$ moderate-intensity rowing session consisted of a $10 \mathrm{~min}$ warm-up to reach each participants' individual target HR (i.e. $70 \%$ of their maximal HR [HRmax], using the equation 220-age as their HRmax), followed by a 20 min plateau phase of constant aerobic activity, rowing at their individual target $\mathrm{HR}$, followed by a $5 \mathrm{~min}$ power interval phase (5 sets of ten consecutive pulls at maximum effort performed at the beginning of each minute, followed by regular intensity strokes for the remainder of each minute) and finally, a 5 min active cool-down period where rowing was continued at a slow pace. Data (rowing distance and session duration) 
were recorded on an individual logcard for each training session. Participants were advised to organize their training sessions on alternate days. The initial 1-3 sessions took place at a different site (Rothschild Hospital, Paris) to study visits, to maintain investigator blinding. These individualized sessions were carried out by a trained physiotherapist to ensure feasibility, to teach participants the ExP, how to use the HR monitor, the rowing machine (Concept $2^{\mathrm{TM}}$ ) and how to perform the rowing movement. Once the physiotherapist and participant were satisfied that the ExP could be performed unsupervised at home, the rowing machine was then delivered to the participant's home for the duration of the intervention period ( 3 months). The Concept $2^{\mathrm{TM}}$ rowing machine was selected as it is a stationary ergometer where one is seated and simultaneously exercises upper and lower limbs and the trunk thus targeting the multiple muscles which can be affected in MG. Adherence was defined as having completed at least 20 (frequency) $30 \mathrm{~min}$ (duration) sessions.

\subsection{Outcomes}

The primary endpoint was the adjusted mean betweengroup difference in MG-specific HRQoL, assessed with the French version of the Myasthenia Gravis Quality of Life questionnaire (total score) [19] (MGQOL-15-F; range 0-60; higher scores represent lower perceived HRQoL) between the ExP and UC group from M3 (baseline) to M6 (postintervention).

To assess tolerability of the ExP compared to UC, adverse events were recorded at each monthly visit except serious adverse events such as $\mathrm{MG}$ crisis or $\mathrm{MG}$ exacerbation with hospitalization which were notified immediately. MG crisis was defined as rapid clinical deterioration necessitating either non-invasive or invasive mechanical ventilation. MG exacerbation was defined as clinical deterioration with beyond usual impact on daily life, with or without modification of medications. Tolerance was evaluated monthly by assessing cardiorespiratory status (cardiac and thoracic auscultation, vital signs, electrocardiogram, cardiothoracic pain, dyspnea, laboratory tests (serum hematology, creatine kinase), muscular/articular pain (selfreport), and medication dosage (corticosteroids and/or AChEi) modification.

Prespecified secondary efficacy endpoints included the adjusted mean between-group difference between the ExP compared to UC from M3 to M6 in: MG symptom severity evaluated with the quantitative Myasthenia Muscle Score [20] (MMS; range 0-100; lower scores represent greater symptom severity), impact of MG on daily function, evaluated with the Myasthenia Gravis Activities of Daily Living scale [21] (MG-ADL; range 0-24, higher scores represent greater impact of $\mathrm{MG}$ on function), physical capacity evaluated with the 6 min walk test (6MWT) for walking endurance [22,23], maximal voluntary isometric strength of elbow flexion and knee extension using a Biodex (Shirley, USA) and handgrip strength using a MyoGrip and respiratory function (Forced Vital Capacity, maximal inspiratory and expiratory pressures evaluated using a Vitalograph spirometer and a MicroRPM). In addition, change in non-specific QoL (WHOQOL Bref) [24,25] and psychological status; depression (Beck Depression Inventory) [26], anxiety (State Trait Anxiety Inventory) [27] and self-esteem (Self-Esteem Inventory scale) [28] before and after the ExP were assessed. Exploratory endpoints consisting of cytokine levels of immunological markers, IL-6 and TNF- $\alpha$ were evaluated between M3 and M6.

\subsection{Statistical analysis}

Sample size calculations were described previously [17]. The primary analysis was conducted in the intention-to-treat (ITT) population, where all randomized participants were analyzed in the group they were allocated to irrespective of any protocol deviation. The primary outcome was analyzed using analysis of covariance (ANCOVA), with changes in MGQOL-15-F as the dependent variable, and randomization group, MGQOL-15-F score at baseline and center as covariates. A similar model was used for secondary and exploratory outcomes defined as changes from M3. For binary secondary outcomes, absolute risk difference and relative risks were estimated. For the monthly MMS and MG-ADL scores, statistical tests were based on linear mixed models on the ranked scores, with random subject intercept and slope, and a separate fixed-effect for each time as well as interactions with the randomization arm from M4 onwards, to force arms to be similar at randomization and to attribute any observed difference to random variation. Magnitude of treatment effect was estimated by differences in least-squares group means in mixed-effect models with bootstrap confidence interval. Cytokines were analyzed after log-transformation and adjusted ratios of geometric means are presented. For values under the lower limit of detection, ANCOVA was replaced by tobit models, with the same formulation. For safety analyses, the numbers of participants with at least one AE or SAE were compared by Fisher's exact tests, and the number of AEs by Poisson regression. Missing data were handled by multiple imputation by chained equations. In addition to baseline subject characteristics, the imputation model considered height, weight, MGQOL-15-F, MMS, MGADL, WHO-QOL, STAI, Beck, MINI, SEI, 6 min walking distance (6MWD), and adherence status in the intervention arm at M3, M6 and M9. Imputations were performed separately in each randomization group as recommended [29]. Imputation of continuous variables was performed using predictive mean matching. Forty imputed datasets were generated and analyzed. Results were then pooled. Physiological (e.g. strength), biological (cytokines) and safety outcomes were not imputed. No allowance was made for multiplicity for secondary endpoints. All tests were twotailed with $\mathrm{p}$ values less than 0.05 defined as significant. A compliers average causal effect (CACE) analysis was carried out using an instrumental variable approach. Notification of AEs and visit tracking was done electronically. 


\section{Recruitment}

\section{Enrolment}

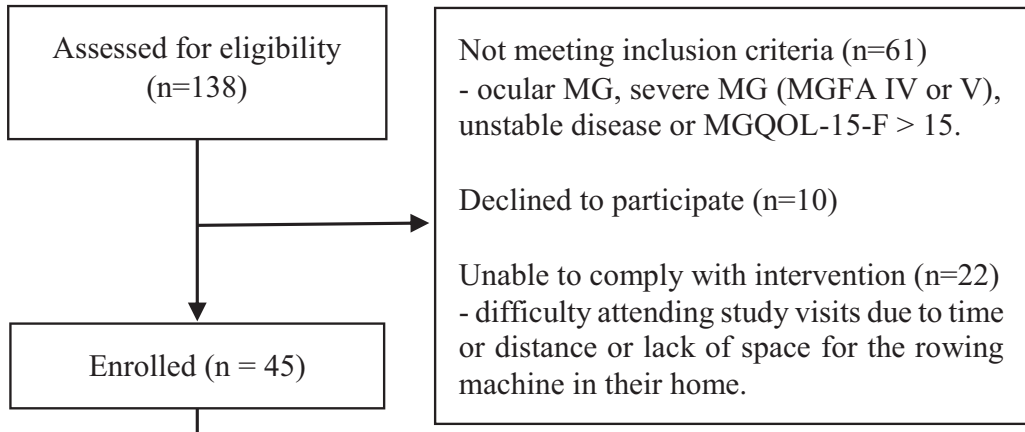

Excluded ( $\mathrm{n}=2)$ as unable to comply with intervention

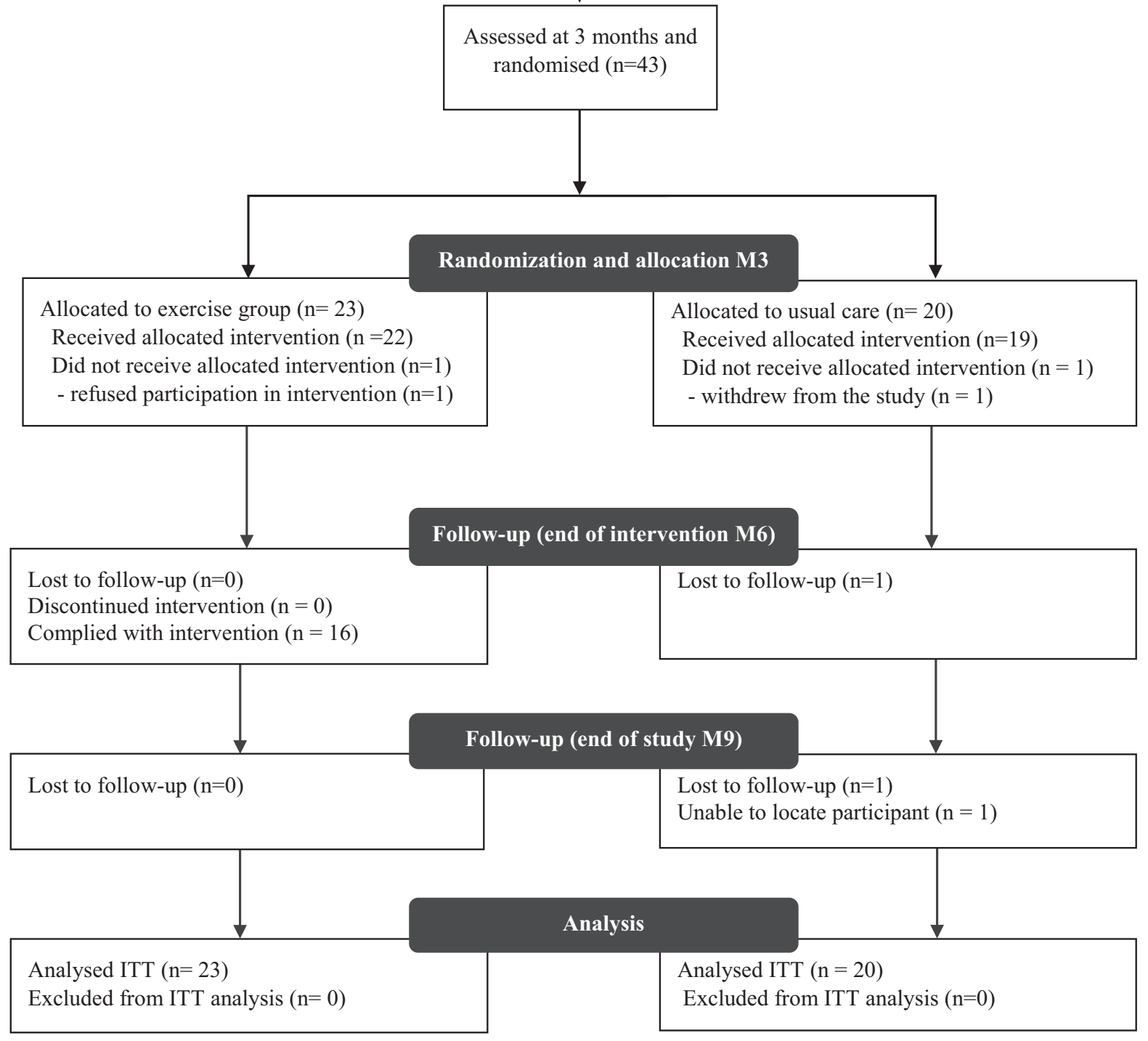

Fig. 1. Participant flow CONSORT diagram. ITT: intention-to-treat. 
Table 1

Demographic, disease characteristics, medical history and activity of participants at baseline i.e. all untrained ${ }^{\mathrm{a}}$.

\begin{tabular}{|c|c|c|c|}
\hline & All $(n=43)$ & Exercise $(n=23)$ & Control $(n=20)$ \\
\hline Mean age, years & 45.5 (10.0) 29 to 70 & $47.1(9.0) 34$ to 64 & 43.7 (10.9) 29 to 70 \\
\hline Female & $40(93 \%)$ & $23(100 \%)$ & $17(85 \%)$ \\
\hline Mean body mass index $\left(\mathrm{kg} / \mathrm{m}^{2}\right)$ & $28.4(5.5) 20.1$ to 45.4 & 27.1 (4.7) 20.1 to 38.8 & 29.9 (6.0) 20.6 to 45.4 \\
\hline $\mathrm{BMI} \geq 30$ & $13(32 \%)$ & $5(24 \%)$ & $8(40 \%)$ \\
\hline \multicolumn{4}{|l|}{ MG subtype (MGFA class) } \\
\hline Class IIa/Class IIb & $17(40 \%) / 6(14 \%)$ & $9(39 \%) / 4(17 \%)$ & $8(40 \%) / 2(10 \%)$ \\
\hline Class IIIa/Class IIIb & $14(33 \%) / 6(14 \%)$ & $5(22 \%) / 5(22 \%)$ & $9(45 \%) / 1(5 \%)$ \\
\hline \multicolumn{4}{|l|}{ Autoantibody profile } \\
\hline AChR & $35(81 \%)$ & $20(87 \%)$ & $15(75 \%)$ \\
\hline MuSK & $3(7 \%)$ & $1(4 \%)$ & $2(10 \%)$ \\
\hline Seronegative for $\mathrm{AChR}$ and MuSK & $5(12 \%)$ & $2(9 \%)$ & $3(15 \%)$ \\
\hline History of the disease & $7(16 \%)$ & $6(26 \%)$ & $1(5 \%)$ \\
\hline \multicolumn{4}{|l|}{ MG onset } \\
\hline \multicolumn{4}{|l|}{ Juvenile, $<18$ years } \\
\hline Early, $18-50$ years & $30(70 \%)$ & $13(57 \%)$ & $17(85 \%)$ \\
\hline Late, $>50$ years & $6(14 \%)$ & $4(17 \%)$ & $2(10 \%)$ \\
\hline Mean disease duration, years & 14.3 (11.0) 1.3 to 44.5 & 17.6 (13.0) 1.3 to 44.5 & 10.4 (6.6) 2.1 to 25.9 \\
\hline Thymectomy & $28(65 \%)$ & $18(78 \%)$ & $10(50 \%)$ \\
\hline \multicolumn{4}{|l|}{ Treatment at randomization } \\
\hline AchE inhibitor & $37(86 \%)$ & $19(83 \%)$ & $18(90 \%)$ \\
\hline Glucocorticoid & $18(42 \%)$ & $10(43 \%)$ & $8(40 \%)$ \\
\hline Prednisone dose (mg) & $3.2(4.3)$ & $3.2(4.3)$ & $3.1(4.4)$ \\
\hline Immunosuppressor & $28(65 \%)$ & $13(57 \%)$ & $15(75 \%)$ \\
\hline \multicolumn{4}{|l|}{ Comorbidities } \\
\hline Thyroid dysfunction & $7(16 \%)$ & $4(17 \%)$ & $3(15 \%)$ \\
\hline Osteoporosis & $10(23 \%)$ & $6(26 \%)$ & $4(20 \%)$ \\
\hline Hypertension & $8(19 \%)$ & $5(22 \%)$ & $3(15 \%)$ \\
\hline Type 2 diabetes mellitus & $4(9 \%)$ & $2(9 \%)$ & $2(10 \%)$ \\
\hline Accelerometer data & 37 & 22 & 15 \\
\hline Days worn & & $6[5$ to 7$]$ & $7[5$ to 7$]$ \\
\hline Average hours worn per day & & $14.2(0.8)$ & $14.5(0.8)$ \\
\hline Minimum hours worn per day & & $13.1(12$ to 15$)$ & $13.2(12$ to 16$)$ \\
\hline Maximum hours worn per day & & $15.3(14$ to 16$)$ & $15.5(14$ to 16$)$ \\
\hline $\begin{array}{l}\text { Average activity (count per minute } \\
\text { per day) }\end{array}$ & & 446 (355 to 553$)$ & 566 (354 to 696$)$ \\
\hline
\end{tabular}

per day)

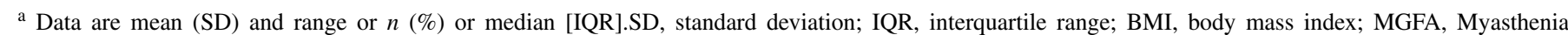

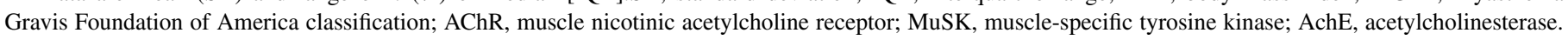

\section{Results}

\subsection{Participants}

Fig. 1 presents the study flow diagram. Between October 3rd, 2014 and July 7th, 2017, 138 patients were screened, of which 45 were enrolled in the study with 23 being randomly assigned to the exercise intervention and 20 to the UC control group. Forty-one participants (91\%) completed the study. Two participants withdrew before randomization. One participant refused to undertake the ExP but remained in the study. Two participants (control group) were lost to follow-up, one following randomization (M3) and the other in their last month of the study (between M8 and M9). Baseline characteristics are presented in Tables 1,2.

\subsection{Intervention adherence}

Of the 23 participants randomized to the ExP, a mean of 24 (range 0-38) forty-minute sessions were completed. Sixteen (70\%) participants adhered to the ExP (Table S3). Reasons for non-adherence were mainly work commitments. Reasons for missing occasional sessions included: the flu, weekend away, menstrual pain/tiredness. Participant characteristics were similar between adherers and non-adherers (Table S4).

\subsection{Primary endpoint (Intention-to-treat)}

There was an adjusted between-groups mean difference of -0.8 points $(95 \% \mathrm{CI}-5.4$ to 3.7 ) on the MGQOL-15-F score at the end of the intervention period (M6) and an adjusted between-groups mean difference of 3.9 points $(95 \% \mathrm{CI}-1.7$ to 9.5) at follow-up (M9) (Table 2), indicating that there was no difference in MG-QOL-15-F score between the two groups at either time point.

\subsection{Exercise tolerance}

Two participants from the control group necessitated hospitalization for an acute MG exacerbation. No exacerbation was reported in the ExP group throughout the entire study (Tables 3,4). There was no between-groups 
Table 2

Intention-to-treat analysis of the primary outcome. Analyses of secondary outcomes ${ }^{\mathrm{a}}$.

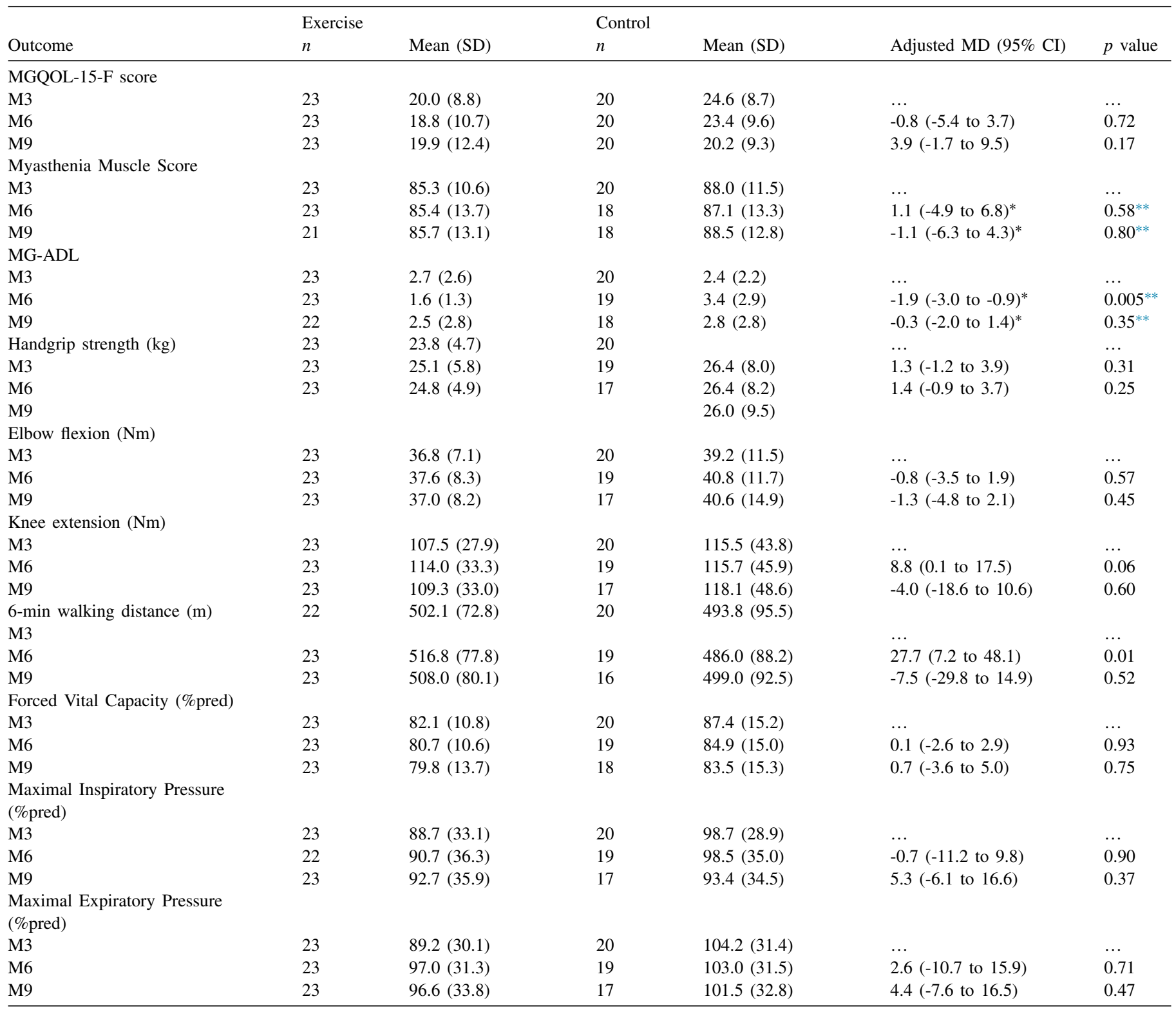

${ }^{a}$ Analyses of imputed data of primary efficacy endpoint (MGQOL-15-F: French version of the 15-item MG-specific quality of life scale). Secondary efficacy endpoints; symptom severity (Myasthenia Muscle Score), limb strength, walking capacity (six-minute walking distance), pulmonary function (Forced Vital Capacity, Maximal inspiratory pressure, Maximal expiratory pressure). \%pred, \% predicted; SD, standard deviation; MD, mean difference; $95 \%$ CI: 95\% Confidence Interval; M3, Randomization, at month 3; M6, end of intervention period, at month 6; M9, end of study at month 9. *Adjusted mean difference estimated by a latent class mixed-effect models, and $95 \%$ confidence interval by bootstrap

** p-value obtained by rank ANCOVA.

difference with regards to exercise tolerance including cardiorespiratory indicators, muscular or articular pain (Table 3), nor concerning non serious adverse events such as infection, allergic reaction, cephalalgia (Table 4). There was no evidence of raised creatine phosphokinase levels with exercise (Table S5).

\subsection{Secondary endpoints}

There was a significant adjusted between-groups mean difference of -1.9 for the MG-ADL (95\%CI -3.0 to -0.9 ) and $27.7 \mathrm{~m}$ for the $6 \mathrm{~min}$ walking distance $(95 \% \mathrm{CI} 7.2$ to 48.1) in favor of the ExP group at M6 but these differences were not maintained at M9 (Table 2). Knee extension strength increased in the ExP group without reaching statistical significance $(8.8 \mathrm{Nm}, \% 95 \mathrm{CI} 0.1$ to 17.5$)$, Table 2. No between-groups difference was observed for the MMS, elbow or grip strength, pulmonary function, general QoL (WHOQOL BREF) or psychological status (STAI, BDI-13, SEI) (Table 2, Table S6). Further, there was no between-groups difference in prednisone dose $-0.4(95 \% \mathrm{CI}-2.1$ to 1.2$)$ nor AChEi dose -0.1 (95\% CI -0.6 to 0.4$)$ at M6, nor at M9 for 
Table 3

Analysis of tolerance to exercise based on neurologist assessment at each monthly visit.

\begin{tabular}{|c|c|c|c|c|c|c|c|}
\hline & $\begin{array}{l}\text { Exercise } \\
n\end{array}$ & No. $(\%)$ & $\begin{array}{l}\text { Control } \\
n\end{array}$ & No. $(\%)$ & $\mathrm{RD}(95 \% \mathrm{CI})$ & RR $(95 \%$ CI $)$ & $p$ value \\
\hline \multicolumn{8}{|l|}{ MG Exacerbations } \\
\hline No. (\%) until M6 & 23 & $0(0)$ & 19 & $3(16)$ & $-16 \%(-38$ to 2$)$ & $0(0$ to 0.98$)$ & 0.084 \\
\hline No. (\%) until M9 & 23 & $0(0)$ & 18 & $5(28)$ & $-28 \%(-51$ to -7$)$ & $0(0$ to 0.55$)$ & 0.011 \\
\hline \multicolumn{8}{|l|}{$\begin{array}{l}\text { Cardiac, respiratory, articular and } \\
\text { muscular symptoms up to M6 }\end{array}$} \\
\hline Cardio-thoracic pain - no. $(\%)$ & 19 & $6(32)$ & 14 & $2(14)$ & $17 \%(-13$ to 42$)$ & $2.21(0.62$ to 8.84$)$ & 0.42 \\
\hline ECG abnormalities - no. $(\%)$ & 21 & $3(14)$ & 17 & $0(0)$ & $14 \%(-6$ to 35$)$ & $\infty(0.70$ to $\infty)$ & 0.24 \\
\hline Dyspnea exertional - no. $(\%)$ & 23 & $5(22)$ & 19 & $7(37)$ & $-15 \%(-40$ to 12$)$ & $0.59(0.23$ to 1.51$)$ & 0.32 \\
\hline Articular pain & 20 & $9(45)$ & 17 & $11(65)$ & $-20 \%(-46$ to 12$)$ & $0.70(0.37$ to 1.27$)$ & 0.32 \\
\hline Muscular pain & 20 & $11(55)$ & 17 & $11(65)$ & $-10 \%(-37$ to 21$)$ & $0.85(0.49$ to 1.48$)$ & 0.74 \\
\hline
\end{tabular}

RD: absolute risk difference; RR: relative risk; 95\% CI: 95\% Confidence Interval; M6, month 6; M9, month 9.

Table 4

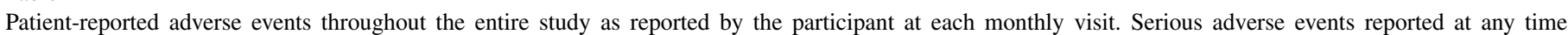
throughout the study and declared to the sponsor.

\begin{tabular}{|c|c|c|c|}
\hline Variable & $\operatorname{Exercise}(n=23)$ & $\operatorname{Control}(n=20)$ & $p$ value \\
\hline No. of adverse events* & 31 & 31 & 0.58 \\
\hline Allergic reaction & 1 & 1 & \\
\hline Cardiac disorder & 1 & 0 & \\
\hline Cephalalgia & 3 & 1 & \\
\hline Infection or infestation & 5 & 6 & \\
\hline Musculoskeletal symptoms & 6 & 13 & \\
\hline MG worsening & 2 & 2 & \\
\hline Respiratory symptoms & 3 & 0 & \\
\hline Other & 10 & 8 & \\
\hline$\geq 1$ event $-\mathrm{n}(\%)$ & $16(70)$ & $14(70)$ & $>0.99$ \\
\hline \multicolumn{4}{|l|}{ Serious adverse events } \\
\hline Death or life-threatening event - n $(\%)$ & $0(0)$ & $0(0)$ & $>0.99$ \\
\hline Disability or incapacity - n (\%) & $0(0)$ & $0(0)$ & $>0.99$ \\
\hline Hospitalization - n (\%) & $0(0)$ & $2(10)$ & 0.21 \\
\hline $\begin{array}{l}\text { Hospitalization for exacerbation of myasthenia gravis } \\
-\mathrm{n}(\%)\end{array}$ & $0(0)$ & $2(10)$ & 0.21 \\
\hline Other significant event $-\mathrm{n}(\%)$ & $0(0)$ & $0(0)$ & $>0.99$ \\
\hline
\end{tabular}

62 adverse events were declared for 30 patients, among which 2 were considered a serious adverse event. $P$ values based on Fisher's exact tests except

* where $P$ value is based on Poisson regression. $n$, number of participants.

prednisone $-0.2(95 \% \mathrm{CI}-1.5$ to 1.0$)$ or, $\mathrm{AChEi}-0.0(95 \% \mathrm{CI}$ -0.5 to 0.4 ).

\subsection{Exploratory endpoints}

There was no between-groups difference in adjusted ratios of geometric means from baseline in cytokines IL-6; 1.09 (95\% CI 0.97 to 1.23 ) nor TNF- $\alpha$; 0.97 (95\% CI 0.86 to 1.10 ), at M6, nor at M9, IL-6; 1.07 (95\%CI 0.95 to 1.21$)$, TNF- $\alpha$; $1.02(95 \%$ CI 0.91 to 1.13$)$.

\section{Discussion}

This randomized, clinical trial of individuals with mild-moderately severe, generalized and stabilized MG, demonstrated that a 3-month unsupervised home exercise program did not improve HRQoL (MGQOL-15-F) compared to UC. The exercise program was, however, well tolerated, with improvement in the perceived impact of MG on daily life
(MG-ADL score) and walking capacity (6MWD), without a between-group difference in psychological or immunological markers.

We believed that HRQoL would be an appropriate primary endpoint for measuring the pluripotent effects of exercise on both physical and psychological domains. Quality of life measures provide information with regards to how satisfied one is with their current level of functioning compared to what they perceive to be possible or ideal [30]. However, self-perceived HRQoL is a complex construct integrating multiple different dimensions and is subject to a response shift phenomenon whereby one's internal reference can change over time or as a result of an intervention (herein exercise), or placebo [31]. We were not able to assess to what extent this phenomenon occurred. It is possible that the absence of a significant change in MGQOL-15-F was due to the intervention itself. The frequency, mode and duration of the exercise program may not have been sufficient to shift this complex construct nor, to improve psychological 
status. As reported elsewhere [17], we initially planned to include patients with an MGQOL-15-F score above 30 in order to have a greater chance of detecting a benefit from exercise. It appeared that for the majority of patients with an MGQOL-15-F score > 30, MG was not stabilized, which we considered a contra-indication to the moderate-intensity exercise intervention.

The improvement of walking endurance evaluated with the 6MWT and trend toward an increase in knee extension strength indicates that our exercise program had an impact on muscle performance, further confirmed by the CACE analyses (Table S8). Importantly, this demonstrates a transfer from a rowing exercise to walking capacity, which we did not specifically train. One may argue that an increase of $27.7 \mathrm{~m}$ over six minutes is small however, it is not only clinically relevant [32] but it should be noted that the distance walked in this cohort was high prior to randomization $(498.1 \pm 83.4 \mathrm{~m})$, making it more difficult to have such a significant change [33]. Given that our exercise intervention was aerobic, these effects are likely due to improvements in muscle metabolism and efficiency. Improvement in cardiorespiratory fitness may also have occurred as we found significant cardiorespiratory gains demonstrated by an increase in rowing distance for the same target heart rate.

Lower limb strength and functional gains (30 s chair stand test) have been demonstrated in previous studies of exercise interventions in $\mathrm{MG}[7,8]$. We would like to emphasize that any gain in strength for MG patients is important as weakness likely contributes to fatigue and reduced daily function [34]. Consistent with our results, although the training programs in these studies included upper limb training, no improvements were observed in the upper limb. These studies were withinsubject control (contralateral limb tested) [7] and repeated measures design [8], each consisting of 11 participants. Thus it was important to confirm findings in a methodologically rigorous randomized, controlled trial with a larger cohort. Further, our exercise intervention was unsupervised and there was no specific strength training as per the other two protocols $[7,8]$.

Tolerance and safety were thoroughly assessed throughout this study via clinical examinations, vital parameters as well as biological indicators. One of the major findings in this clinical trial is the tolerance of the exercise program. We did not observe any MG deterioration nor increased muscular or articular pain or, cardiorespiratory symptoms compared to UC. This is likely to be related to our choice of a moderateintensity program with specific training in using the rowing machine and the individualized nature of the program. We are not able to determine to what extent the high tolerance option may have compromised efficiency. Only a trial comparing an exercise program with various intensities and durations could address this issue. However, good tolerance is a major argument for recommending this type of exercise program in a larger population. The rowing machine is a popular device for home exercise as it is simple to learn and perform.

When choosing an unsupervised, home-based intervention there is greater risk of poor adherence than a supervised programme. Adherence should be taken into account when generalization is considered. Seven (30\%) participants were considered as non-adherers. Their reported reasons were unrelated to the exercise program nor to their MG and baseline characteristics between the two groups were similar (Table S4). The non-adherence in our study is considered acceptable considering the intervention was home-based and unsupervised and prior studies have shown similar compliance for exercise interventions [35]. Our definition of non-adherence was arbitrary and the small number of participants limits comparison between adherers and nonadherers, in determining the minimal exercise dosage for efficacy. This RCT was designed with intention-to-treat (ITT) analyses however, CACE analyses provides further insight into the effects of the exercise according to compliance. The difference in the results of the CACE and ITT analysis suggests that it would be worth optimizing adherence of the exercise intervention in future studies. This could possibly be achieved with direct supervision or with remote monitoring using wearables, keeping in mind that device adherence would also need to be taken into consideration.

This study presented some methodological limitations. Whilst our sample size could be considered modest, there was an a priori calculated sample size, which is comparable to other studies of exercise in rare disease [36,37]. Despite the fact that MG is more prevalent in women before the age of 50 (juvenile and early MG represents $86 \%$ of this cohort) [38], there still remains disproportionately more women (40) than men (3) in this study, and all men were randomly assigned to the control group. We note that this sex imbalance was also present in a previous study suggesting possible selection bias which should be taken into account in future studies of exercise interventions in MG [7]. We acknowledge that our results should be confirmed in a larger population, notably to ensure the applicability and tolerance of our exercise program particularly in men whom were largely lacking in this trial. Whilst it would be of interest to assess whether the effects of exercise varies according to specific clinical or immunological phenotypes of MG, our aim was to first evaluate the tolerance in all types of MG. Due to the type of intervention, blinding of participants was not possible and some improvement could be related to positive expectation bias particularly for those who were motivated to perform exercise [39].

At this stage more research is required to answer questions concerning the type of exercise, intensity, duration and frequency and the impact of each aspect on individuals and subgroups of MG. It may be important to consider current lifestyle and physical activity levels for better tailoring of an exercise intervention. In addition, we selected patients with stabilized MG. Therefore, the feasibility, tolerance and effectiveness of exercise in non-stabilized MG will need to be specifically assessed. Strengths of this study include the rigorous study design and the comprehensive assessments of the functional, immunological and psychological impact of exercise using validated measures. Participant retention throughout the study was good with only two participants (control group) withdrawing. 


\section{Conclusion}

This randomized clinical trial with intention-to-treat analyses is the first to demonstrate that a 3-month, homebased unsupervised exercise program is well tolerated and does not contribute to exacerbations in adults with stabilized, gMG. This is a major result as benefit and risk of exercise is a major concern for individuals with $\mathrm{MG}$ and for which no specific recommendations are currently available. Further research is necessary to evaluate different dosages of exercise and their effects, in this population.

\section{Funding}

This study was sponsored by the Assistance Publique Hôpitaux de Paris «Direction de la Recherche Clinique et de l'Innovation". This study was funded through a grant from the Programme Hospitalier de Recherche Clinique PHRC régional 2012 (Ministère de la Santé AOR12149), in collaboration with the Institute of Myology. The funders had no role in the design of the study; nor data analysis, data interpretation, nor in the writing of this manuscript.

\section{Trial registration:}

ClinicalTrials.gov identifier NCT02066519.

\section{Disclosure of Conflicts of Interest}

S. Birnbaum, R. Porcher, B. Clair, S. Demeret, B. Eymard, M. Gargiulo, E. Louët, S. Berrih-Aknin, R. Le Panse, P. Aegerter, J.Y. Hogrel, T. Sharshar report no disclosures relevant to the manuscript.

\section{Data availability}

Individual de-identified participant data may be shared for research/academic purposes once all secondary analyses have been completed. Requests for access to data must be made to the corresponding author and sponsor and should be accompanied by a methodologically sound proposal. A signed data sharing agreement is required before access can be provided.

\section{Authors' contributions}

See Table S9 in supplementary information.

\section{Declaration of Competing Interest}

The authors declare that they have no known competing financial interests or personal relationships that could have appeared to influence the work reported in this paper.

\section{Acknowledgments}

We sincerely thank all participants. We thank the APHP for the grant through the French Ministry of Health grant program for Clinical Research (AOR12149). We also thank the Institute of Myology for their financial contribution. We acknowledge the assistance of Sophie Hue, Mathieu Surenaud, IMRB luminex platform, Creteil. We would also like to acknowledge the nurses and administration staff who played a crucial role in this study.

\section{MGEX Study group}

Sylvie Azerad, Guillaume Bassez, Anthony Behin, Sonia Berrih-Aknin, Francis Bolgert, Nawal Derridj Ait-Younes, Yasmine Domingo, Mélinée Frenkian, Diane Friedman, Asmaa Jobic, Pascal Laforêt, Isabelle Ledoux, Judith Mendelson, Sandra Misdrahi, Cécilia Orblin Bedos, Benjamin Rohaut, Jacques Ropers, Elodie Soler, Philippe Thoumie, Frédérique Truffault, Nicolas Weiss, Linda William.

\section{Supplementary materials}

Supplementary material associated with this article can be found, in the online version, at doi:10.1016/j.nmd.2021.05. 002 .

\section{References}

[1] Sanders DB, Wolfe GI, Benatar M, Evoli A, Gilhus NE, Illa I, et al. International consensus guidance for management of Myasthenia Gravis: executive summary. Neurology 2016;87:419-25.

[2] Barnett C, Herbelin L, Dimachkie MM, Barohn RJ. Measuring clinical treatment response in Myasthenia Gravis. Neurol Clin 2018;36:339-53.

[3] Boldingh MI, Dekker L, Maniaol AH, Brunborg C, Lipka AF, Niks EH, et al. An up-date on health-related quality of life in myasthenia gravis -results from population based cohorts. Health Qual Life Outcomes 2015;13:115.

[4] Bankole LC, Millet GY, Temesi J, Bachasson D, Ravelojaona M, Wuyam B, et al. Safety and efficacy of a 6-month home-based exercise program in patients with facioscapulohumeral muscular dystrophy: a randomized controlled trial. Medicine 2016;95:e4497.

[5] Siciliano G, Schirinzi E, Simoncini C, Ricci G. Exercise therapy in muscle diseases: open issues and future perspectives. Acta Myol Myopathies Cardiomyopathies Off J Mediterr Soc Myol 2019;38:233-8.

[6] Cheng A, Morsch M, Murata Y, Ghazanfari N, Reddel SW, Phillips WD. Sequence of age-associated changes to the mouse neuromuscular junction and the protective effects of voluntary exercise. PLoS ONE 2013;8:e67970.

[7] Lohi EL, Lindberg C, Andersen O. Physical training effects in Myasthenia Gravis. Arch Phys Med Rehabil 1993;74:1178-80.

[8] Westerberg E, Molin CJ, Sporndly Nees S, Widenfalk J, Punga AR. The impact of physical exercise on neuromuscular function in Myasthenia gravis patients: a single-subject design study. Medicine 2018;97:e11510.

[9] Wong SH, Nitz JC, Williams K, Brauer SG. Effects of balance strategy training in myasthenia gravis: a case study series. Muscle Nerve 2014;49:654-60.

[10] Rahbek MA, Mikkelsen EE, Overgaard K, Vinge L, Andersen H, Dalgas U. Exercise in myasthenia gravis: a feasibility study of aerobic and resistance training. Muscle Nerve 2017;56:700-9.

[11] Westerberg E, Molin CJ, Lindblad I, Emtner M, Punga AR. Physical exercise in myasthenia gravis is safe and improves neuromuscular 
parameters and physical performance-based measures: a pilot study. Muscle Nerve 2017;56:207-14.

[12] Schuch FB, Vancampfort D, Rosenbaum S, Richards J, Ward PB, Stubbs B. Exercise improves physical and psychological quality of life in people with depression: a meta-analysis including the evaluation of control group response. Psychiatry Res 2016;241:47-54.

[13] Stubbs B, Vancampfort D, Rosenbaum S, Firth J, Cosco T, Veronese N, et al. An examination of the anxiolytic effects of exercise for people with anxiety and stress-related disorders: a meta-analysis. Psychiatry Res 2017;249:102-8.

[14] Herring MP, O'Connor PJ, Dishman RK. The effect of exercise training on anxiety symptoms among patients: a systematic review. Arch Intern Med 2010;170:321-31.

[15] Kruger K, Mooren FC, Pilat C. The Immunomodulatory effects of physical activity. Curr Pharm Des 2016;22:3730-48.

[16] Gleeson M, Bishop NC, Stensel DJ, Lindley MR, Mastana SS, Nimmo MA. The anti-inflammatory effects of exercise: mechanisms and implications for the prevention and treatment of disease. Nat Rev Immunol 2011;11:607-15.

[17] Birnbaum S, Hogrel JY, Porcher R, Portero P, Clair B, Eymard B, et al. The benefits and tolerance of exercise in myasthenia gravis (MGEX): study protocol for a randomized controlled trial. Trials 2018;19:49.

[18] Boutron I, Moher D, Altman DG, Schulz KF, Ravaud P. Extending the CONSORT statement to randomized trials of nonpharmacologic treatment: explanation and elaboration. Ann Intern Med 2008;148:295-309.

[19] Birnbaum S, Ghout I, Demeret S, Bolgert F, Eymard B, Sharshar T, et al. Translation, cross-cultural adaptation, and validation of the French version of the 15-item Myasthenia Gravis quality of life scale. Muscle Nerve 2017;55:639-45.

[20] Sharshar T, Chevret S, Mazighi M, Chillet P, Huberfeld G, Berreotta C, et al. Validity and reliability of two muscle strength scores commonly used as endpoints in assessing treatment of myasthenia gravis. J Neurol 2000;247:286-90.

[21] Wolfe GI, Herbelin L, Nations SP, Foster B, Bryan WW, Barohn RJ. Myasthenia gravis activities of daily living profile. Neurology 1999;52:1487-9.

[22] Enright PL. The six-minute walk test. Respir Care 2003;48:783-5.

[23] Salci Y, Karanfil E, Balkan AF, Kutukcu EC, Ceren AN, Ayvat F, et al. Functional exercise capacity evaluated by timed walk tests in myasthenia gravis. Muscle Nerve 2019;59:208-12.

[24] Skevington SM, Lotfy M, O'Connell KA. The World Health Organization's WHOQOL-BREF quality of life assessment: psychometric properties and results of the international field trial. A report from the WHOQOL group. Qual Life Res Int J Qual Life Asp Treat Care Rehabil 2004;13:299-310.
[25] Leplege A, Reveillere C, Ecosse E, Caria A, Riviere H. Propriétés psychométriques d'un nouvel instrument d'évaluation de la qualité de vie, le WHO-QOL-26, à partir d'une population de malades neuro-musculaires. L'Encephale 2000;16:13-22.

[26] Collet L, Cottraux J. [The shortened Beck depression inventory (13 items). Study of the concurrent validity with the Hamilton scale and Widlocher's retardation scale]. Encephale 1986;12:77-9.

[27] Bruchon-Schweitzer M, Paulhan I, manuel du Le. STAI-Y de CD Spielberger, Adaptation Française. Paris: ECPA; 1993.

[28] Coopersmith S. Inventaire D'estime de Soi. Paris: Edition du Centre de Psychologie Appliquée; 1984.

[29] Sullivan TR, White IR, Salter AB, Ryan P, Lee KJ. Should multiple imputation be the method of choice for handling missing data in randomized trials? Stat Methods Med Res 2016:962280216683570.

[30] Cella DF, Tulsky DS. Measuring quality of life today: methodological aspects. Oncology (Huntingt) 1990;4:29-38 discussion 69.

[31] Bonnetain F, Fiteni F, Efficace F, Anota A. Statistical challenges in the analysis of health-related quality of life in cancer clinical trials. J Clin Oncol 2016;34:1953-6.

[32] Baert I, Freeman J, Smedal T, Dalgas U, Romberg A, Kalron A, et al. Responsiveness and clinically meaningful improvement, according to disability level, of five walking measures after rehabilitation in multiple sclerosis: a European multicenter study. Neurorehabil Neural Repair 2014;28:621-31.

[33] Salci Y, Karanfil E, Fil Balkan A, Calik Kutukcu E, Ceren AN, Ayvat F, et al. Functional exercise capacity evaluated by timed walk tests in Myasthenia Gravis. Muscle Nerve 2019;59:208-12.

[34] Dobkin BH. Fatigue versus activity-dependent fatigability in patients with central or peripheral motor impairments. Neurorehabil Neural Repair 2008;22:105-10.

[35] Kelley GA, Kelley KS. Dropouts and compliance in exercise interventions targeting bone mineral density in adults: a meta-analysis of randomized controlled trials. J Osteoporos 2013;2013:250423.

[36] Bello-Haas VD, Florence JM, Kloos AD, Scheirbecker J, Lopate G, Hayes SM, et al. A randomized controlled trial of resistance exercise in individuals with ALS. Neurology 2007;68:2003-7.

[37] Gellen B, Messonnier LA, Galacteros F, Audureau E, Merlet AN, Rupp $\mathrm{T}$, et al. Moderate-intensity endurance-exercise training in patients with sickle-cell disease without severe chronic complications (EXDRE): an open-label randomized controlled trial. Lancet Haematol 2018;5:e554-62.

[38] Berrih-Aknin S, Le Panse R. Myasthenia Gravis: a comprehensive review of immune dysregulation and etiological mechanisms. J Autoimmun 2014;52:90-100.

[39] Sackett DL. Why did the randomized clinical trial become the primary focus of my career? Value Health 2015;18:550-2. 